\title{
The effect of orientation in binocular contour rivalry of real images and afterimages*
}

\author{
N. J. WADE \\ University of Dundee, Dundee DDI $4 H N$, Scotland
}

\begin{abstract}
Binocular rivalry was investigated using gratings of different orientations in three experiments. No consistent effects of orientation were found for predominance measures of rivalry between real images. Rivalrous afterimages, on the other hand, did exhibit orientation selectivity: vertical gratings were visible for longer than were 45-deg gratings. This effect was compared to the similar orientation selectivity found for monocular observation of grating afterimages. Comparisons of binocular rivalry between real images and afterimages were made in terms of the frequency distributions of the dominance periods.
\end{abstract}

Contours of different orientations presented to corresponding areas of each eye undergo phases of apparent dominance and suppression. This is referred to as binocular rivalry. For example, if a vertical line is presented to one eye and a horizontal line to the other, rivalry will take place at their intersection such that alternately one line and then the other will appear to be unbroken. When a grating rather than a single line is presented to each eye, dominance is indexed by the visibility of the contours of one orientation alone. In this sense, gratings are more suitable stimuli for the investigation of contour rivalry, as dominance is readily discriminable; with single lines, a wide variety of fragmentations can occur, and dominance may be difficult to determine (see Cogan \& Goldstein, 1972). An attendant feature of rivalry between gratings is that composites, in which different orientations dominate in different parts of the field, are also visible for some of the time.

The measures most commonly employed in rivalry experiments are the rate of alternation and the overall or percentage duration for which one stimulus is dominant (predominance). The predominance of a given stimulus can be influenced by features such as the amount of contour, the contrast at the contour, and its clarity (see Levelt, 1968). The purpose of the present experiments was to determine whether or not contour orientation could influence predominance in binocular rivalry. The first experiment examined rivalry between a vertical ( $0 \mathrm{deg})$ grating and a variety of inclined ones; the gratings were observed as real images during continuous stereoscopic viewing. Two further experiments compared rivalry between 0 - and 45-deg gratings, viewed as prolonged afterimages and also as real images. Binocular afterimages, being perfectly stabilized, are not influenced by eye movements during observation.

*This research was supported by a grant from the Science Research Council. I wish to thank Kirsteen Greig for her assistance in the collection and analysis of the data. I am grateful to J. Cormack for conducting the optical examinations of the Ss in Experiment III.
Moreover, monocular afterimages of gratings are affected by orientation (Wade, 1973), and the experiments sought to determine whether similar effects occurred during binocular rivalry.

\section{EXPERIMENT I}

Almost all previous experiments on bincoular contour rivalry have used lines that were orthogonal. In this experiment, a range of orientations was employed. One eye was presented with a vertical grating and the other with an inclined one, the orientation of which could be varied between 15 and $90 \mathrm{deg}$ to the vertical. The gratings were in the form of circles so that they could be completely superimposed for all the orientations used. The overall durations for which each of the gratings in a given rivalry configuration were visible in their entirety (i.e., their predominance) were measured.

\section{Method}

\section{Subjects}

Twenty Ss from an introductory course in psychology participated in the experiment. None of the Ss were practiced in observing binocular rivalry. All had normal or corrected vision.

\section{Apparatus}

The stimulus configurations consisted of square-wave gratings of 8.9 cycles/deg spatial frequency and a contrast of 0.7 . They were in the form of circles subtending $1 \frac{1}{4}$ deg at the eye. The gratings were viewed in a stereoscope, and the cards bearing the configurations could be adjusted to achieve superimposition. Two microswitches, operated by S's right and left hands, were connected to timers which recorded the cumulative duration of switch depressions.

\section{Procedure}

All rivalry configurations consisted of a vertical grating presented to one eye and an inclined one to the other. The inclined gratings were oriented at $15,30,45,60,75$, or 90 deg clockwise of the vertical. Each member of the six pairs of rivalrous stimuli was presented to each eye to balance any effects of eye dominance. The task of S was to depress one switch when the vertical grating alone was visible and the other switch when only the inclined grating could be seen. The switches were 
Table 1

Mean Predominance Durations (Seconds) for Vertical and Inclined Gratings Viewed for 100 Sec Under Conditions of Binocular Rivalry, Together With the Standard Deviations

\begin{tabular}{lcccccccccccc}
\hline & \multicolumn{10}{c}{ Grating Orientation (Degrees) } \\
\cline { 2 - 15 } & 0 & 15 & 0 & 30 & 0 & 45 & 0 & 60 & 0 & 75 & 0 & 90 \\
\hline Mean & 25.1 & 35.2 & 26.3 & 28.1 & 28.1 & 28.3 & 28.2 & 29.4 & 28.6 & 29.0 & 30.0 & 25.1 \\
SD & 18.4 & 19.2 & 15.1 & 15.0 & 15.7 & 14.3 & 15.3 & 14.7 & 17.6 & 14.4 & 17.3 & 13.8 \\
\hline
\end{tabular}

depressed for the duration of visibility of these categories, and neither switch was depressed when composites were apparent. A practice trial was given to ensure that the instructions had been understood. There were 12 trials each of $100 \mathrm{sec}$ duration, with an interval between them of $60 \mathrm{sec}$, during which S's eyes remained closed. The order of presentation of the rivalrous pairs was randomized.

\section{Results and Discussion}

The data for a given pair of rivalrous stimuli were averaged over eyes to balance any effects due to eye dominance. The mean overall durations for which each member of a rivalrous pair was visible (predominance) are shown in Table 1 , together with the standard deviations. For all configurations, the duration for which either grating was predominant was approximately $60 \mathrm{sec}$ of the $100-\mathrm{sec}$ inspection period. The remaining time would have comprised composites. The mean durations are very similar for rivalry between 0 -deg gratings and those from 30 to $75 \mathrm{deg}$. The 0 -deg grating was visible for slightly longer than the 90-deg and much shorter than the 15-deg grating. Six t tests were computed for the differences between the mean visibility durations of the vertical and inclined gratings for each rivalry configuration. The differences failed to reach statistical significance in every case $(p>.05)$, indicating that a vertical grating is visible for about the same duration as an inclined one in rivalry with it, irrespective of its orientation.

Contour orientation does not seem to influence predominance in binocular rivalry. The longer visibility of the $15 . \mathrm{deg}$ grating relative to the vertical one may have been due to some degree of central fusion in addition to slight cyclofusional rotation of the eyes (see Kertesz \& Jones, 1970). The fused percept would have appeared midway between the two orientations, i.e., nonvertical, and this could have inflated the 15 -deg predominance measure. This effect was not produced by all Ss, and the individual variations were large.

The only condition favoring the vertical contours involved rivalry with a horizontal grating. Such a difference might be expected from the pattern of nonconjugate eye movements that occur during rivalry, since these are mainly in a horizontal direction (Kaufman, 1963). That is, a small horizontal eye movement would displace the contours of a vertical grating onto new retinal areas, whereas a horizontal grating would be displaced such that only the ends of the contour would project onto new retinal areas.
However, the vertical grating was not predominant for significantly longer than the horizontal in this experiment.

\section{EXPERIMENT II}

The effects of contour displacements over the eyes can be eliminated by binocular stabilization of the rivalrous stimuli. This was done in the present experiment by means of prolonged afterimages, and the pattern of rivalry was compared with that for equivalent real image stimuli. Any differences that emerge from these patterns might be related to the effects of eye movements in binocular rivalry.

Prolonged afterimages of gratings viewed monocularly exhibit orientation selectivity: vertical afterimages remain visible in their entirety for longer than do those at $45 \mathrm{deg}$ (Wade, 1973). The same orientations were chosen for study in this experiment in order to determine whether or not such orientation selectivity also obtained in binocular rivalry.

The temporal characteristics of rivalrous 0 - and 45-deg gratings viewed as afterimages and real images were examined. The predominances and frequencies of visibility of each grating were measured under both conditions. In addition, the visibility of composites was recorded in the same manner as was dominance.

\section{Method}

\section{Subjects}

Twenty-four Ss, graduate and undergraduate members of the University of Dundee, took part in the experiment. All had normal or corrected vision.

\section{Apparatus}

The gratings were viewed through a modified prism stereoscope in front of which was a chinrest and forehead support for controlling S's head position. The gratings were located 28.5 in. from S's eyes and were formed from apertures in black masking cards. The apertures corresponded to square-wave gratings of 2 cycles/deg spatial frequency; they were in the form of a circle with an angular substense of $3 \mathrm{deg}$. The gratings were either 0 or $45 \mathrm{deg}$, and they could be located on corresponding retinal areas by means of adjustable biprisms. Three switches were positioned in front of $\mathbf{S}$; these activated timers and separate channels of a Rustrak event recorder.

Afterimages were generated by the discharge of a Multiblitz Report flash grun, with an output of $120 \mathrm{~J}$ in $2 \mathrm{msec}$. The real images were produced by transillumination through the apertures, with a luminance of approximately $20 \mathrm{fL}$. Since the 
Table 2

Mean Predominance Durations (Seconds) and Frequencies of Visibility for the 0 and 45-Deg Gratings and for Composites Under Conditions of Rivalry Between Real Images and Afterimages, Together With the Standard Deviations

\begin{tabular}{|c|c|c|c|c|c|c|c|}
\hline \multirow{2}{*}{$\begin{array}{l}\text { Visibility } \\
\text { Category }\end{array}$} & & \multicolumn{3}{|c|}{ Real Images } & \multicolumn{3}{|c|}{ Afterimages } \\
\hline & & 0 Deg & Composite & $45 \mathrm{Deg}$ & 0 Deg & Composite & $45 \mathrm{Deg}$ \\
\hline Duration & $\begin{array}{l}\text { Mean } \\
\text { SD }\end{array}$ & $\begin{array}{l}26.9 \\
10.2\end{array}$ & $\begin{array}{l}33.8 \\
20.1\end{array}$ & $\begin{array}{l}31.9 \\
11.8\end{array}$ & $\begin{array}{r}41.5 \\
9.2\end{array}$ & $\begin{array}{l}9.9 \\
8.8\end{array}$ & $\begin{array}{r}35.3 \\
8.7\end{array}$ \\
\hline Frequency & $\begin{array}{l}\text { Mean } \\
\text { SD } \\
\end{array}$ & $\begin{array}{r}16.7 \\
6.9 \\
\end{array}$ & $\begin{array}{r}14.6 \\
6.8 \\
\end{array}$ & $\begin{array}{r}16.8 \\
6.8 \\
\end{array}$ & $\begin{array}{r}10.6 \\
3.6\end{array}$ & $\begin{array}{l}5.7 \\
4.3\end{array}$ & $\begin{array}{r}10.5 \\
3.8\end{array}$ \\
\hline
\end{tabular}

gratings were in the form of a circle and the apertures provided the only source of light in the otherwise dark room, superimposition of the real images was readily maintained without additional aids to binocular alignment.

\section{Procedure}

A brief description and demonstration of binocular rivalry was given to $S$, followed by the instructions regarding switch depressions. The task involved depressing the right switch for the visibility of vertical lines alone, the left one for the 45-deg lines, and the center switch for the composites. Four trials with the 0and 45-deg rivalrous gratings were given, two with afterimages and two with real images, in order to balance the orientations over eyes. The order of presentation of the trials was counterbalanced over Ss, with the constraint that the two afterimage and the two real image rivalry conditions be successive. In the afterimage condition, $\mathrm{S}$ was given a practice trial and then dark-adapted for $10 \mathrm{~min}$ before the first afterimage trial was started. Following completion of the first trial, a further 5-min dark adaptation was given before the second afterimages were generated. A dim light illuminated the stimuli prior to the flash to ensure that the gratings were superimposed. The afterimages were observed with the eyes closed and in darkness. For rivalry between real images, $S$ aligned the gratings before commencement of a trial, and they remained in that position throughout the trial. The inspection period was $100 \mathrm{sec}$ for all conditions.

\section{Results and Discussion}

The predominance durations and frequencies were averaged over eyes to reduce any eye dominance effects. The means and standard deviations for all measures are given in Table 2. The total duration of visibility of any category was about $93 \mathrm{sec}$ for real images and $87 \mathrm{sec}$ for afterimages. In the case of the former, it is possible that the latencies involved in so many responses (48 on average) contributed to the 7 -sec difference between the visibility and the observation periods. For afterimages, there were fewer responses ( 27 on average), and so this is unlikely to have been the main factor. However, both afterimages were noted to disappear occasionally during the inspection period, and a similar effect has been noted in rivalry between optically stabilized patterns (Ditchburn \& Pritchard, 1960); furthermore, the afterimages had faded completely for some Ss before termination of the $100-\mathrm{sec}$ inspection period. These factors render a direct comparison between rivalry under the two conditions difficult. The striking differences are that rivalry is much slower with afterimages: the average period of dominance (predominance duration/frequency) is in the order of $4 \mathrm{sec}$ for afterimages and about $2 \mathrm{sec}$ for real images. Breese (1899) noted a similar effect for rivalry between much weaker afterimages than those generated here. Moreover, composites are visible for considerably shorter overall durations with afterimages than with real images.

Grating orientation appeared to have a differential effect on rivalry under the two conditions: the 45-deg grating was visible for slightly longer with real images, whereas the 0-deg grating was predominant for longer with rivalry between afterimages. The data for each rivalry condition were analyzed separately. For real image rivalry, neither the duration nor the frequency measures of the three visibility categories differed significantly $(p>.05)$. In the afterimage condition, the durations of visibility differed significantly $[F(2,42)=$ $69.27, \mathrm{p}<.001]$. Post hoc comparisons using the method devised by Rodger (1965) indicated that the difference between the 0 - and 45-deg means was significant $(p<.05)$, and also that the composite duration differed significantly from the iverage of the 0 and 45-deg durations $(p<.001)$. The frequency measure yielded overall significance $[F(2,42)=18.06, p<.001)$, and this was due mainly to the significant comparison between the composite frequency and that for the average of the 0 - and 45-deg frequencies $(p<.001)$.

The results with real images support those of Experiment $I$ in yielding no effect of orientation on predominance in binocular rivalry. Moreover, the duration values correspond quite closely with those of the previous experiment; in both, predominance of either orientation accounted for abolit $60 \%$ of the inspection period.

Rivalrous afterimages, on the other hand, exhibit orientation selectivity-the 0 -deg grating predominates for significantly longer than the 45-deg grating. This effect corresponds to the selectivity for monocularly viewed grating afterimages (Wade, 1973). They also yield very short durations of composites relative to the predominance of either rivalry configuration alone.

\section{EXPERIMENT III}

This experiment was concerned with the same general problem as the previous one, but a smaller number of Ss was tested intensively. In addition to examining contour rivalry between gratings viewed as real images and 
Table 3

Mean Predominance Durations (Seconds) and Frequencies of Visibility of 0 - and 45-Deg Gratings and Composites for Rivalry of Real Images and Afterimages*

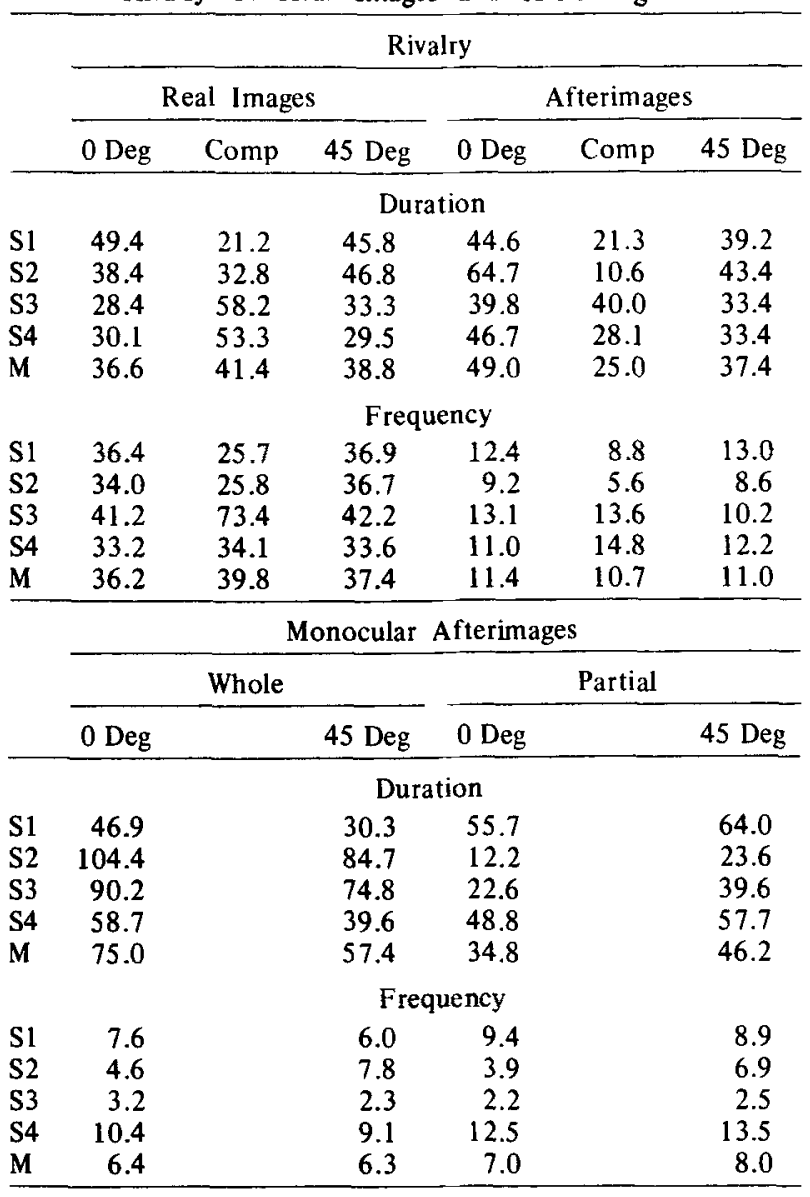

*The whole and partial durations and frequencies for monocular afterimage visibility are also presented.

afterimages, the monocular visibilities of afterimages were also studied. By presenting each orientation singly to each eye, it is possible to determine whether or not the degree of orientation selectivity evident for monocular afterimages is equivalent to that produced in rivalry.

\section{Method}

\section{Subjects}

Four Ss, two male and two female, were tested in the experiment. They were well practiced in observing binocular rivalry. Two Ss were emmetropic in one eye and had slight uncorrected myopia in the other; one was emmetropic in both eyes, and the fourth had corrected myopia in one eye while the other was emmetropic. None of the Ss had any evidence of astigmatism.

\section{Apparatus}

The apparatus was the same as that used in Experiment II. The gratings had the same overall angular subtense ( $3 \mathrm{deg}$ ), but the spatial frequency was 4 cycles/deg. The orientations of the gratings were 0 and $45 \mathrm{deg}$. In the monocular conditions, one field of the stereoscope was blanked out. The luminance of the gratings for the real image rivalry condition was again $20 \mathrm{fL}$.

\section{Procedure}

Each $\mathrm{S}$ was given eight sessions for afterimages and eight for rivalry between real images. The afterimage sessions consisted of two binocular rivalry trials and four monocular trials in which each orientation was presented to each eye. The rivalry trials followed the same procedure as those in Experiment II, except that the observation period was $120 \mathrm{sec}$. The predominance durations and frequencies for each grating were recorded as well as those for composites. For the monocular trials, $\mathrm{S}$ was instructed to depress one switch when the afterimage was visible in its entirety and another switch when it was only partially visible throughout the $120-\mathrm{sec}$ inspection period. The order of presentation of the six trials was balanced so that the binocular pairs were either first or last and the eye used in the monocular conditions was alternated. A 10-min dark-adaptation period was given before the first trial, and the intertrial interval was $5 \mathrm{~min}$.

For rivalry between real images, $S$ was presented with two trials only per session, and responded in terms of the visibility of either grating or a composite. The inspection period was also $120 \mathrm{sec}$.

\section{Results and Discussion}

The data for each grating orientation were averaged over eyes, and the results for each $\mathrm{S}$ are given in Table 3 , together with the means. During binocular rivalry, the total visibility duration of either grating or a composite was about $117 \mathrm{sec}$ for real images and $111 \mathrm{sec}$ for afterimages. In both conditions, these visibility durations occupied a larger proportion of the inspection period than those in Experiment II. The monocular afterimages were visible in either whole or partial form for about $110 \mathrm{sec}$ for the 0 -deg and $104 \mathrm{sec}$ for the 45 -deg gratings. The average dominance periods for rivalrous afterimages were about $4 \mathrm{sec}$, which is in good agreement with Experiment II. In fact, in both experiments, the dominance periods for the 0-deg afterimages were longer than those for the $45-\mathrm{deg}$ afterimages. The dominance periods of the gratings in real image rivalry were about $1 \mathrm{sec}$.

The results for rivalry between real images correspond closely with those of the previous two experiments: predominance duration was not significantly influenced by grating orientation $(p>.05)$. The values, when converted to percentages to equate the inspection periods, are very similar to those of the previous experiment. The frequencies of visibility were not affected by orientation ( $p>.05$ ), although these values are much higher than in Experiment II. This was due, most probably, to the difference in the spatial frequencies of the gratings used in the experiments. That is, small eye movements during observation would be likely to cross more contour boundaries with the higher than with the lower spatial frequency gratings. It should be noted that all Ss reported some difficulty in following the rapid pattern of changes in the present experiment. 
The results for rivalry between afterimages provide general support for those of Experiment II, although the variation between the Ss was quite large. The mean predominance of the 0-deg grating differed significantly from that for the 45-deg visibility $[\mathrm{t}(3)=3.19, \mathrm{p}<.05]$. The orientation selectivity was about $10 \%$ compared to $6 \%$ in Experiment II. The durations of visibility of composites were even more variable, and did not differ significantly from the average visibility of the two gratings $(p>.05)$. The differences between the frequencies of visibility were not significant $(p>.05)$.

The monocular afterimages exhibited orientation selectivity: the 0-deg grating was visible in whole form for significantly longer than the 45-deg grating [ $t(3)=$ $16.68, \mathrm{p}<.001]$. Contrariwise, the partial visibility of the 45-deg grating was significantly longer than that for the 0-deg grating $[t(3)=5.75, p<.05]$. Neither of these effects was accompanied by frequency differences $(p>.05)$. These results are similar to those reported previously (Wade, 1973).

Therefore, orientation selectivity has been shown to operate both for monocular grating afterimages and in their rivalrous combination. Moreover, the relationships between the predominance of the 0- and 45-deg gratings in rivalry and in monocular observation are very similar. If the ratios of the 0 - to the $45-\mathrm{deg}$ durations are calculated for each condition, the average values for both are about 1.3. This indicates that a similar degree of orientation selectivity is operating for monocular observation and in binocular rivalry.

\section{GENERAL DISCUSSION}

Binocular contour rivalry between gratings viewed as real images is not influenced by orientation. A vertical grating remains visible for a similar period to an inclined one. Rivalry between afterimages, on the other hand, exhibits orientation selectivity favoring the vertical orientation. That is, a vertical grating afterimage predominates for significantly longer than an inclined one. The extent of this selectivity corresponds to that for the monocular visibility of similar afterimages (Experiment III). The difference in the effect of orientation on rivalry between real images and between afterimages might be related to the occurrence of small eye movements in the former, but it is by no means clear how this masking would have been produced. Nonconjugate eye movements have a greater horizontal than vertical amplitude (Kaufman, 1963), and such would appear to favor the visibility of vertical contours during normal stereoscopic observation.

The shorter average dominance periods for rivalry between real images than between afterimages can be more readily interpreted, due to the movement of the images over the eyes in the former. The mean dominance periods (predominance/frequency) were about $4 \mathrm{sec}$ in each experiment using afterimages, but between 1 and $2 \mathrm{sec}$ for real images. These values correspond to those
LEFT EYE

RIGHT EYE

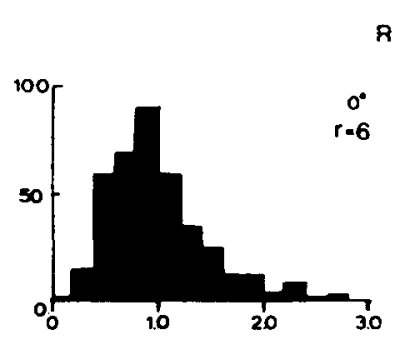

REAL IMAGES
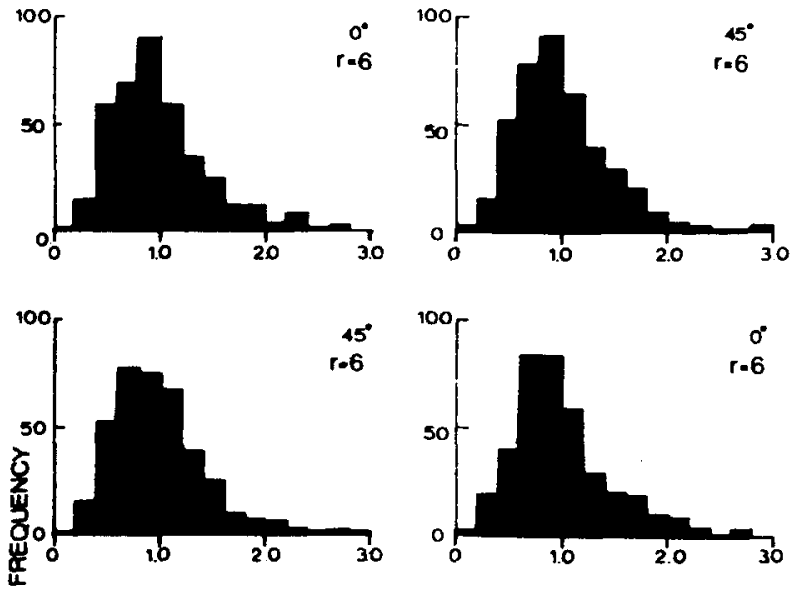

AFTERIMAGES
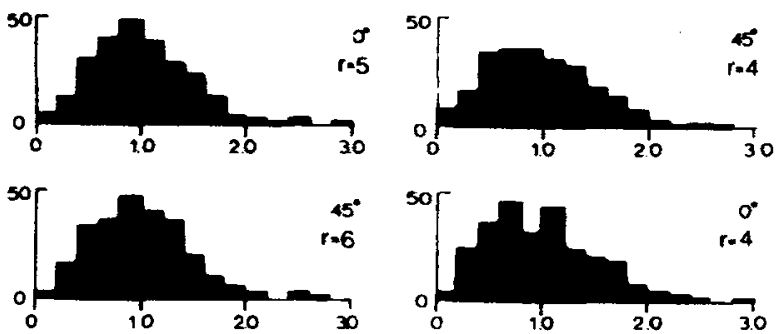

DURATION (SET TO COMMON BASE)

Fig. 1. Frequency distributions of dominance periods for Experiment II under all rivalry conditions. The data for each $S$ were divided by their own mean duration so that the overall mean for all distributions is $\mathbf{1 . 0 0}$.

obtained by Blake, Fox, and McIntyre (1971) and Fox and Herrmann (1967) for ring-disk rivalry involving afterimages and real images, respectively. In order to investigate the stochastic properties of rivalry, they examined the dominance periods of their alternation data by means of a runs test, autocorrelation, and the frequency distribution. The stimuli they used exhibited virtually no composites. The occurrence and measurement of composites in the present investigation precludes the use of the first two tests, but it is possible to plot the frequency distributions of the dominance periods in the various conditions. Following the procedure employed by both Levelt (1968) and Fox and Herrmann (1967), the individual dominance periods were measured for each $S$, and these were then divided by the mean duration for that $S$, resulting in an overall mean value of 1.00 . This procedure was followed for each condition in Experiments II and III, and the resulting frequency distributions are shown in Figs. 1 and 2. The usefulness of fitting a specific function to these distributions can be questioned. Nonetheless, it is 
LEFT EYE REAL IMAGES
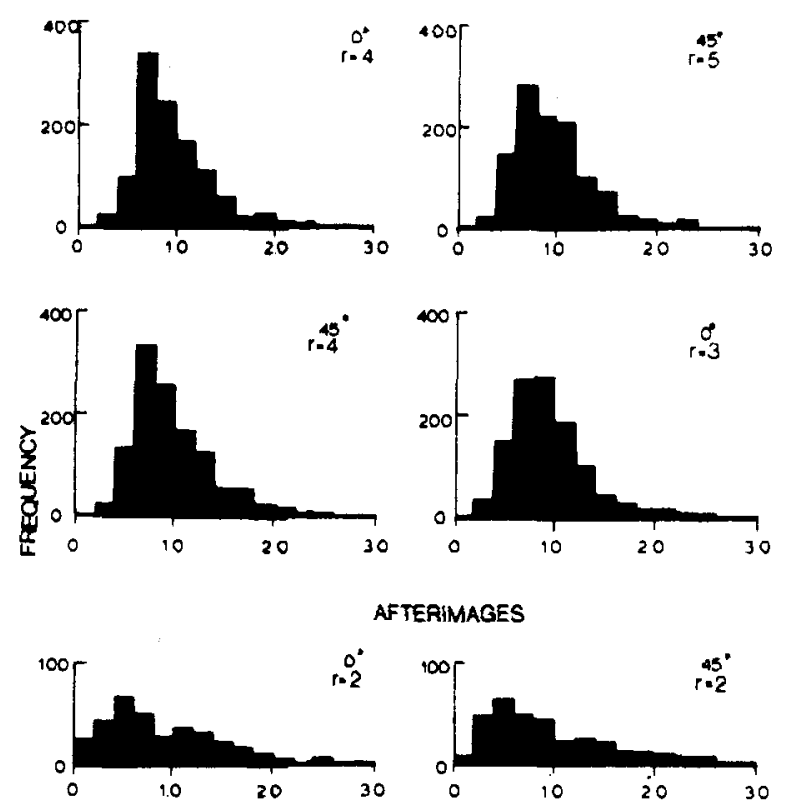

AFTEAIMAGES
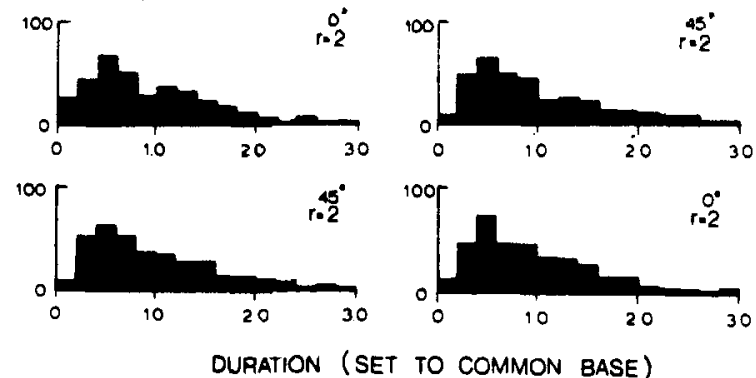

Fig. 2. Frequency distributions of dominance periods for all rivalry conditions in Experiment III. The frequency scale for the afterimage conditions has been duubled relative to that for real images.

of interest to note that all the distributions are positively skewed and conform to the general shape of the gamma function, which function also approximated the distributions of Levelt's (1968) and Fox's (Blake et al, 1971; Fox \& Herrmann, 1967) data. Furthermore, the $r$ parameter of the gamma distributions for rivalry between real images are in close correspondence with those reported previously. There are, however, some revealing differences in the $r$ values for rivalry between afterimages and real images: $r$ is smaller for the afterimage than for the real image conditions. In Experiment II, the average $r$ for the real image distributions was 6 while that for the afterimages was 5 . In Experiment III, where more extensive data were derived from a smaller number of $S$ s so that the estimates of $\mathrm{r}$ were more precise, the corresponding values were 4 and 2 . Since the $r$ parameter can be interpreted as an index of the number of component events comprising a response (Restle, 1961), then clearly more such components would be involved in rivalry between real images than between afterimages. These would most probably include the eye movements that occur under normal stereoscopic observation. The influence that incorporation of the composite category has had on the above points remains unknown, but this issue might be resolved by examining rivalry between short single lines viewed either as real images or as afterimages.

The orientation selectivity that has been demonstrated for monocular afterimages and rivalrous afterimages corresponds to a wide variety of similar perceptual effects (see Appelle, 1972). The occurrence of such selectivity in binocular rivalry further supports the cortical localization of these effects. The absence of any effects of orientation during rivalry between real images might have been due to the pattern of small eye movements during observation. These might also have been involved in the rapid changes in dominance for real images, and in the high proportion of composite visibility. Afterimages alternated more slowly and composites comprised a small proportion of the total visibility duration.

\section{REFERENCES}

Appelle, S. Perception and discrimination as a function of stimulus orientation. Psychological Bulletin, 1972, 78, 266-278.

Blake, R. R., Fox, R., \& McIntyre, C. Stochastic properties of stabilized-image binocular rivalry alternations. Journal of Experimental Psychology, 1971, 88, 327-332.

Breese, B. B. On inhibition. Psychological Monographs, 1899, 3, No. 1.

Cogan, R., \& Goldstein, A. G. Reporting of fragmentations in the binocular rivalry of contours. American Journal of Psychology, 1972, 85, 569-584.

Ditchbum, R. W., \& Pritchard, R. M. Binocular vision with two stabilized retinal images. Quarterly Journal of Experimental Psychology, 1960, 12, 26-32.

Fox, R., \& Herrmann, J. Stochastic properties of binocular rivalry alternations. Perception \& Psychophysics, 1967, 2, 432-436.

Kaufman, L. On the spread of suppression and binocular rivalry. Vision Research, 1963, 3, 401-415.

Kertesz, A. E., \& Jones, R. W. Human cyclofusional response. Vision Research, 1970, 10, 891-896.

Levelt, W. J. M. On binocular rivalry. The Hague: Mouton, 1968.

Restle, F. Psychology of judgment and choice: A theoretical essay. New York: Wiley, 1961.

Rodger, R. S. Intermediate statistics. Sydney: University Cooperative Bookshop, 1965.

Wade, N. J. Orientation and spatial frequency effects on linear afterimages. Perception \& Psychophysics, 1973, 13, 446-450.

(Received for publication March 7, 1973; revision received August 27, 1973.) 